\title{
BMJ Open Examining the effectiveness of a web- based intervention for symptoms of depression and anxiety in college students: study protocol of a randomised controlled trial
}

Eirini Karyotaki, ${ }^{1,2}$ Anke M Klein, ${ }^{3}$ Heleen Riper, ${ }^{1,2}$ Leonore de Wit, ${ }^{1,2}$ Lisa Krijnen, ${ }^{1,2}$ Eline Bol, ${ }^{1,3}$ Felix Bolinski, ${ }^{1,2}$ Simone Burger, ${ }^{1,2}$ David D Ebert, ${ }^{1,4}$ Randy P Auerbach, ${ }^{5}$ Ronald C Kessler, ${ }^{6}$ Ronny Bruffaerts, ${ }^{7}$ Neeltje Batelaan, ${ }^{8,9}$ Claudia M van der Heijde, ${ }^{10}$ Peter Vonk, ${ }^{10}$ Annet Kleiboer, ${ }^{1,2}$ Reinout W Wiers, ${ }^{3}$ Pim Cuijpers ${ }^{\oplus 1,2}$

To cite: Karyotaki E, Klein AM, Riper $\mathrm{H}$, et al. Examining the effectiveness of a web-based intervention for symptoms of depression and anxiety in college students: study protocol of a randomised controlled trial. BMJ Open 2019;9:e028739. doi:10.1136/ bmjopen-2018-028739

- Prepublication history for this paper is available online. To view these files, please visit the journal online (http://dx.doi. org/10.1136/bmjopen-2018028739).

Received 21 December 2018 Revised 20 March 2019 Accepted 26 March 2019
Check for updates

(C) Author(s) (or their employer(s)) 2019. Re-use permitted under CC BY-NC. No commercial re-use. See rights and permissions. Published by BMJ.

For numbered affiliations see end of article.

Correspondence to

Dr Eirini Karyotaki;

e.karyotaki@vu.nl

\section{ABSTRACT}

Introduction The college years are a peak period for the onset of common mental disorders. Poor mental health is associated with low academic attainment, physical, interpersonal and cognitive impairments. Universities can use online approaches to screen students for mental disorders and treat those in need. The present study aims to assess the effectiveness of a guided web-based transdiagnostic individually tailored intervention to treat students with symptoms of depression and/or anxiety. Methods and analysis The present study is a randomised controlled trial. Participants are Dutch college students ( $\geq 18$ years) with mild to moderate depression and/or anxiety symptoms. The intervention is a guided web-based transdiagnostic individually tailored intervention that targets symptoms of depression and/ or anxiety. The intervention consists of seven online sessions with a duration ranging from 4 to 7 weeks depending on individual progress. A booster session is administered 4 weeks after the completion of the seventh session. Primary outcome measures are the Patient Health Questionnaire for depression and the Generalised Anxiety Disorder 7-item scale for anxiety. These scales are administered at screening, post-treatment and follow-up assessments (6 and 12 months post-randomisation). Ethics and dissemination The Medical Ethics Committee of the Vrije Universiteit Medical Centre has approved the protocol (registration number 2016.583, A2017.362andA2018.421). Results of the trial will be published in a peer-reviewed journal. Trial registration number NTR6797; Pre-results.

\section{INTRODUCTION}

Mental health problems, such as depression and anxiety, have a significant impact on college students' functioning and are notably burdensome. ${ }^{1}$ College years are a peak period for the first onset of common

\section{Strengths and limitations of this study}

- This study aims to advance current knowledge on the effects of web-based interventions in college students with depression and anxiety.

- A transdiagnostic and individually tailored therapeutic approach is employed to target both symptoms of depression and anxiety.

- Both Dutch and International students will be included to increase the generalisability of the findings.

- The power calculation has been based on the primary aims of this study, thereby limiting the power to detect moderators of treatment outcome.

- The assessment of study dropout relies on self-report answers due to privacy restrictions.

mental disorders. ${ }^{2}$ College students experience a variety of stressors (eg, exams, living away from family and financial hardships), which make them prone to mental disorders. Research has shown that depression and anxiety are highly prevalent among college students while the majority of lifetime cases begin before 24 years of age. ${ }^{2}$ Not surprisingly, there is a positive association between mental health and academic attainment. Mental disorders are related to physical, interpersonal and cognitive impairments, which adversely affect educational participation and exam performance. ${ }^{3-5}$ Consequently, there is a high chance of study dropout or delay in higher education, which, in turn, leads to high direct and indirect costs for both individuals and society. ${ }^{67}$

Addressing student mental health might, thus, be effective in improving students' well-being and academic results. However, 
not many college students with depression or anxiety seek or can find help for their condition. Less than $25 \%$ of college students with mental disorders use mental healthcare services. ${ }^{8}$ The university can be an excellent environment for detecting students at high risk of mental disorders and for applying evidence-based treatment approaches to prevent and treat common mental disorders at an early stage. However, the limited resources of college counselling services hamper the detection of students with mental issues. In many universities, psychologists offering services to students treat only study-related problems (eg, exam anxiety and procrastination) and not symptoms of mental disorders, such as depression and anxiety. ${ }^{9}$ In addition, the fear of stigmatisation makes students reluctant to consult university counselling services. ${ }^{10}$ As a result, depression and anxiety are considerably underdiagnosed and typically untreated during college years with an unnecessary chance of aggravation of problems. ${ }^{11}$

The question arises as to how universities and colleges can provide treatment, which is effective, timely, available at low-cost, accessible and that overcomes worries about stigmatisation by maintaining students' anonymity. The Internet can play a crucial role in this endeavour. Presently, internet-based approaches have a high penetration rate and are particularly popular among youth. Many young people with mental disorders seek information on their symptoms online. ${ }^{10}$ Universities can use electronic media to screen for students with mental disorders but also treat those in need. ${ }^{12}$ Recently, web-based psychological interventions have been developed and examined in research and clinical settings. Several randomised controlled trials (RCTs) and meta-analyses have addressed the effectiveness of web-based and other computerised interventions in treating depression and anxiety symptoms. So far, the results have shown that web-based interventions with therapist support are superior to control groups ${ }^{13-17}$ with similar effect sizes to conventional faceto-face treatments. ${ }^{18}$

Furthermore, several studies that examine the effects of web-based transdiagnostic and individually tailored interventions have emerged. ${ }^{19-22}$ Given that depression and anxiety are highly comorbid, interventions aimed at improvement of both depression and anxiety symptoms are needed. Transdiagnostic interventions target common disorder mechanisms, such as avoidance. ${ }^{23}$ Results from a recent meta-analysis showed a medium to large effect size in favour of web-based transdiagnostic/individually tailored interventions compared with controls in treating anxiety $(\mathrm{g}=0.82)$ and depression $(\mathrm{g}=0.79) .{ }^{23}$

Nevertheless, to date, there have been relatively few studies focusing on the effectiveness of web-based interventions in treating college students with depression and/or anxiety disorders. Systematic reviews of technology-based interventions for tertiary students with mental disorders have shown mixed evidence for the effectiveness of technology interventions targeting depression and/or anxiety. ${ }^{1024}$ However, the focus of these reviews was broad; they included studies that employed either prevention or treatment interventions. ${ }^{24-28}$

Similarly, few studies have specifically focused on the effectiveness of transdiagnostic web-based interventions in college students with depression and anxiety. Day et al found that web-based guided transdiagnostic cognitive behavioural therapy (iCBT) is more effective in treating depression $(\mathrm{d}=0.55)$ and anxiety $(\mathrm{d}=0.66)$ compared with a waitlist control in college students. ${ }^{29}$ Moreover, Mullin et al conducted an RCT examining the effects of iCBT in treating anxiety and depression of college students. ${ }^{30}$ The authors found significant results in favour of the transdiagnostic web-based intervention compared with a waiting list (anxiety: $\mathrm{d}=1.33$; depression: $\mathrm{d}=1.59$ ).$^{30}$ Given these encouraging findings, it is important to examine further the effects of these novel therapeutic approaches in treating college students with symptoms of depression and/or anxiety.

\section{Objectives}

Primary objectives

The present study aims to assess the effectiveness of a guided web-based transdiagnostic individually tailored intervention in treating college students with symptoms of depression and/or anxiety.

\section{Secondary objectives}

Additionally, the present study aims to (a) explore participant characteristics as moderators of treatment outcome, (b) examine the acceptability of the treatment and (c) assess whether the investigated intervention prevents university dropout and increases educational achievement.

\section{Hypothesis}

We hypothesise that the interventions will outperform the control condition in reducing depressive and anxiety symptoms of college students.

\section{METHODS AND ANALYSIS}

\section{Trial design}

The present study is a two-arm superiority RCT (1:1 allocation ratio), which compares a guided web-based transdiagnostic individually tailored intervention to treatment as usual (TAU).

\section{Study setting}

The present study is conducted in Dutch universities and colleges. The recruitment of participants and the study procedures are managed by two main centres (the Vrije Universiteit and the Universiteit van Amsterdam).

\section{Eligibility criteria}

Participants are young adults ( $\geq 18$ years) enrolled as bachelor or master students at a university or college in the Netherlands. Students will participate in an online survey, which is part of an epidemiological study assessing the prevalence rates of mental disorders in a college 
student population. This study is embedded within the WHO World Mental Health International College Student initiative (WMH-ICS). Students are invited to participate in the RCT if they: (a) experience mild to moderate depression defined as scoring above the cut-off score of 4 on the Patient Health Questionnaire (PHQ$9)^{31}$ and/or anxiety symptoms defined as scoring above the cut-off score of 4 on the Generalised Anxiety Disorder scale-7 items (GAD-7), ${ }^{32}$ (b) speak Dutch or English fluently and (c) provide written informed consent before participation.

Students are excluded if they: (a) have comorbid bipolar disorder or psychotic disorder according to the Mini-International Neuropsychiatric Interview (MINI), ${ }^{33}$ (b) experience severe depression defined as scoring above the cut-off score of 14 on the PHQ-9 and/or anxiety symptoms defined as scoring above the cut-off score of 14 on the GAD-7 scale, (c) currently receive psychological treatment for depression and/or anxiety or have received treatment in the past 12 months and (d) have slow or no Internet connection (eg, no broadband Internet).

\section{Intervention}

The intervention used in this study, 'ICare Prevent', is a guided web-based transdiagnostic individually tailored intervention with mobile support and is targeted at symptoms of depression and/or anxiety. It can be used on laptops, computers and mobile devices. This intervention has been initially developed in the German language for use in the general population and is based on adaptions of a range of evidence-based interventions. ${ }^{34}{ }^{35}$ Thus, it has been translated into Dutch and English and adapted to college student needs after a series of focus group discussions with college students. ${ }^{36}$ The intervention strategies have been based mainly on cognitive behavioural techniques. It uses text, homework exercises, audio-visual components and information sheets that can be downloaded. Testimonials are used to explain homework.

The participants receive 7 weekly online sessions: (1) introduction into the intervention and its technical aspects, setting goals and importance of pleasant activities, (2) tackling problems and behavioural activation, (3) psychoeducation, (4) cognitive restructuring and challenging negative thoughts, (5) choosing the most prominent symptoms and accordingly for depression: problem-solving; for anxiety: exposure, (6) continuation of strategies selected from session 5 and (7) plan for the future. Four weeks after completion of the seventh session, participants will be invited for a booster session. The individually tailored aspect of the intervention is applied in sessions 5 and 6 . Therein, participants follow disorder-specific exercises by choosing either problem-solving targeted at depressive symptoms or exposure to anxiety-provoking situations, depending on individual preference. On the basis of their personal needs, participants are free to choose elective modules that are integrated into sessions 2-7 (worry and rumination, acceptance of unfulfilled needs, relaxation, alcohol consumption as

\section{Table 1 Intervention content}

Main intervention

\begin{tabular}{|c|c|}
\hline Session & Content \\
\hline 1. Introduction & $\begin{array}{l}\text { Goal setting and importance of } \\
\text { pleasant activities }\end{array}$ \\
\hline 2. Tackling problems & $\begin{array}{l}\text { Identification of problems and } \\
\text { problem-solving based on } \\
\text { behavioural activation }\end{array}$ \\
\hline 3. Psychoeducation & $\begin{array}{l}\text { Psychoeducation focusing } \\
\text { either on depression or anxiety } \\
\text { depending on individual needs }\end{array}$ \\
\hline 4. Cognitive restructuring & $\begin{array}{l}\text { Development of functional } \\
\text { positive thinking after identifying } \\
\text { the relationship between } \\
\text { thoughts, well-being and } \\
\text { practising strategies }\end{array}$ \\
\hline $\begin{array}{l}\text { 5. Choosing most prominent } \\
\text { symptoms }\end{array}$ & $\begin{array}{l}\text { Problem-solving targeted at } \\
\text { either depression or exposure } \\
\text { to anxiety-provoking stimuli } \\
\text { depending on individual needs }\end{array}$ \\
\hline $\begin{array}{l}\text { 6. Deepening of skills chosen } \\
\text { in session } 5\end{array}$ & $\begin{array}{l}\text { Problem-solving and exposure in } \\
\text { daily life }\end{array}$ \\
\hline 7. Plan for the future & $\begin{array}{l}\text { Reflection on goal attainment } \\
\text { and learning experiences. } \\
\text { Implementation of intentions until } \\
\text { the booster session }\end{array}$ \\
\hline
\end{tabular}

8. Booster session ( 4 weeks after the completion of the seventh session)

Reflection on goal attainment and learning experiences. Implementation of intentions during the upcoming months

Elective modules

1. Worry and rumination Information on worry and rumination, using a worrydiary and other techniques to challenge such thoughts

2. Acceptance of unfulfilled needs

Attending to unfulfilled needs and unsolvable problems and learning to accept them

$\begin{array}{ll}\text { 3. Relaxation } & \begin{array}{l}\text { Exercise on progressive muscle } \\ \text { relaxation }\end{array} \\ \begin{array}{l}\text { 4. Alcohol consumption as } \\ \text { emotion regulator }\end{array} & \begin{array}{l}\text { Information on the relationship } \\ \text { between mood and alcohol, self- } \\ \text { assessment of consumption and } \\ \text { techniques to decrease it }\end{array}\end{array}$

5. Self-worth

Information on the effects of low self-worth and exercises to increase it

6. Perfectionism Identifying personal high standards and learning techniques to exit from a vicious circle

7. Appreciation and gratitude Learning how to express gratitude and how to consciously appreciate positive things in daily life

8. Sleep hygiene

Sleep-limitation technique (ie, by initially limiting sleep being able to ultimately sleep better)

emotion regulator, self-worth, perfectionism, appreciation and gratitude, and sleep hygiene). Table 1 gives an overview of the intervention. 
Each session takes between 45 and $60 \mathrm{~min}$. Participants are advised to follow one or maximum two sessions per week. Thus, the total duration of the intervention ranges from 4 to 7 weeks. The online sessions are delivered with written support given by coaches via the messaging function of the intervention platform. Participants are allowed to use the content of the intervention $24 / 7$, as long and as often they want through the online treatment platform. In addition to the online sessions, participants have access to diaries (eg, for tracking positive activities and monitoring sleep), mood graph, homework assignments and the messaging system that allows participants to contact their online coach. The optional mobile app provides access to, for example, diaries. A username and a self-generated password protect participants' access to the intervention.

\section{Online treatment platform}

Minddistrict is the e-health platform hosted by Minddistrict BV, which is an enterprise responsible for the provision and maintenance of the Minddistrict platform. Minddistrict provides the content management system to researchers to upload interventions/questionnaires and to enrol participants/e-coaches. This platform has been repeatedly used by several research projects and routine care services. Minddistrict complies with all European data safety regulations and quality standards.

\section{Support}

Trained psychology master students will deliver support to participants. The training lasts for 1 day and consists of three parts: (a) theory (eg, intervention materials), (b) assignments and (c) practice. Research staff experienced in web-based interventions gives the training. Coaches provide individual manualised asynchronous feedback via the messaging function of the intervention platform after the completion of each module. Moreover, coaches are available to answer additional messages about the treatment content in case they are contacted by the participants at any time point throughout the intervention. The coaches are advised to spend less than $30 \mathrm{~min}$ per individual feedback while the estimated time of feedback is $20 \mathrm{~min}$. Thus, a coach spends in total of approximately 2.5 hours per participant. A senior researcher monitors the feedback written by the coaches to ensure adherence to the treatment protocol.

\section{Treatment as usual}

Participants in the TAU group receive information about the available regular care services in the community, such as help from their general practitioner (GP), primary and secondary mental health services from psychologists/ psychiatrists. These services include mostly medications (eg, antidepressants) and/or low-intensive face-to-face psychotherapies. Students are free to decide whether they would like to seek help or not. Use of these services is recorded through self-report questionnaires at the post-treatment and follow-up assessments. This control condition has been chosen to reflect whether there is a difference between the web-based intervention and what students would normally do.

\section{Primary outcomes}

Participants who will be included in the RCT are assessed by:

\section{Patient Health Questionnaire-9 items}

The PHQ- $9{ }^{37}$ is a self-report outcome measure that can be used to screen depressive symptoms. The PHQ-9 consists of 9 items. Item responses are on a 0-3 scale with total scores ranging from 0 to 27 . Higher scores indicate more severe depression. PHQ-9 shows good psychometric properties with a sensitivity of $0.77(0.71-0.84)$ and a specificity of $0.94(0.90-0.97){ }^{38}$ The PHQ-9 is administered at the screening ( $\mathrm{t} 0)$, post-treatment (t2) and follow-up (t3 and $t 4$ ) assessments in the intervention group.

\section{Generalised Anxiety Disorder scale-7 items}

The 7-item $\mathrm{GAD}^{32}$ scale will be used to measure anxiety symptoms. Each of the 7 items is scored on a $0-3$ scale while total score range is $0-21$. Higher scores indicate more severe anxiety symptoms. The GAD-7 scale shows internal consistency with a value of Cronbach's coefficient $(\alpha)$ ranging from 0.79 to $0.91^{39}$. The GAD-7 is administered at the screening ( 0 0), post-treatment ( $\mathrm{t} 2)$ and follow-up (t3 and t4) assessments.

\section{Mini-International Neuropsychiatric Interview}

The diagnostic interview MINI V.5.0 is conducted via telephone by a trained clinical psychology master student. The MINI is a short-structured interview based on the Diagnostic and Statistical Manual of Mental Disorders, fourth edition, and the International Classification of Diseases criteria. The MINI is used to determine the number of participants with a current/lifetime diagnosis of major depressive disorder, panic disorder, agoraphobia, social phobia and GAD both at the baseline and 12-month follow-up. Moreover, during baseline, the MINI will be used to estimate the number of participants with current/ lifetime diagnosis of major comorbidities (dysthymia, suicidality, [hypo] manic episode, obsessive-compulsive disorder, post-traumatic stress disorder, alcohol dependence/abuse, drug dependence/abuse, psychotic disorders, anorexia nervosa and bulimia nervosa). The MINI shows good psychometric properties with good test-retest reliability and validity. ${ }^{40}$ The MINI is administered at baseline (t1) and 12-month follow-up (t4).

\section{EuroQol—five dimensions}

Quality of life is measured with the EuroQol-five dimensions (EQ-5D). ${ }^{41}$ The EQ-5D is a self-report questionnaire, which measures the health-related well-being for clinical and economic appraisal. More precisely, EQ-5D consists of five items/dimensions: mobility, self-care, ordinary activities, discomfort and mood state, related to anxiety or depression. Each item/dimension consists of three categories ranging from no problems to few and finally to many 
problems. ${ }^{42}$ EQ-5D construct validity is adequate, and this type of measurement can detect meaningful changes for patients with anxiety disorders. EQ-5D is generally consistent with the measure of mood state: depression/anxiety. ${ }^{43}$ The EQ-5D is administered at baseline (t1), post-treatment (t2) and follow-up (t3 and t4) assessments.

\section{Client Satisfaction Questionnaire-8 items}

The Client Satisfaction Questionnaire-8 items (CSQ$8)^{44}$ is used to assess client satisfaction related to the treatment. This self-report questionnaire consists of 8 items. Item responses are on a 1-4 scale with total scores ranging from 8 to 32. Higher scores of CSQ-8 indicate higher satisfaction with the treatment. The CSQ-8 shows high internal consistency with a value of Cronbach's coefficient $(\alpha)$ being $0.93 .{ }^{45}{ }^{46}$ The CSQ-8 is administered at the post-treatment $(\mathrm{t} 2)$

University dropout and educational achievement

University dropout will be monitored through self-report questions administered at post-treatment (t2) and follow-up (t3 and t4) assessments. Regarding educational achievement, the Presenteeism Scale for Students (PSS) is used to assess presenteeism. ${ }^{47}$ The PSS is a valid and reliable measure for the college student population. ${ }^{47}$ Moreover, the students are asked about the number of European Credit Transfer System achieved during a given study period. The educational achievement is measured at the baseline (t1), post-treatment (t2) and follow-up ( $\mathrm{t} 3$ and $\mathrm{t} 4$ ) assessments.

\section{Treatment adherence}

Adherence to treatment is measured by tracking the website usage automatically. Data related to the total number of modules completed, time spent per module and number of logging into the platform are gathered.

\section{Assessments}

Table 2 presents an overview of all measures and assessment points. As mentioned in the Eligibility criteria section, students are recruited through an online survey, which is part of an epidemiological study. In brief, this survey consists of a broad range of short self-administered validated scales assessing mental health problems, such as attention deficit hyperactivity disorder (the Adult Attention Deficit Hyperactivity Disorder Self-Report ${ }^{48}$ ), major

Table 2 Overview of measures and assessment points

$\begin{array}{lllll}\text { Measure (instrument) } & \text { Assessment points } & & \\ & \text { t0 } & \text { t1 } & \text { t3 } & \\ \text { Socio-demographics (e-survey) } & X & & \\ \text { DSM-IV axis I and II disorders (e-survey) } & X & & \\ \text { Daily functioning (e-survey) } & X & \\ \text { Personality traits (e-survey) } & X \\ \text { Clinical measures (e-survey) } & X \\ \text { Suicide plan and/or suicide attempt(s) (e-survey) } & X \\ \text { Childhood maltreatment and domestic violence } & X & & \\ \text { (e-survey) } & & \end{array}$

Academic experiences and functioning, participation $\mathrm{X}$ in athletic and extracurricular activities (e-survey)

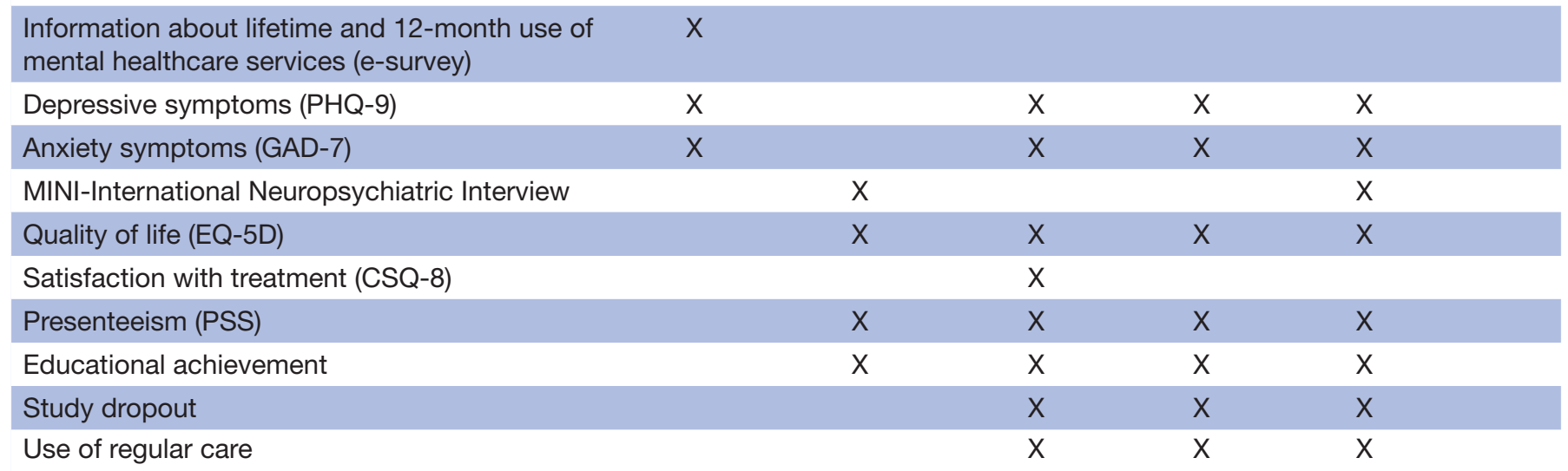

CSQ-8, Client Satisfaction Questionnaire-8 items; DSM-IV, Diagnostic and Statistical Manual of Mental Disorders, fourth edition; EQ-5D, EuroQol-five dimensions; GAD-7, Generalised Anxiety Disorder scale-7 items; PHQ-9, Patient Health Questionnaire-9 items; PSS, Presenteeism Scale for Students; RCT, randomised controlled trial; t0, screening (total cohort); t1, baseline (RCT); t2, post-treatment (RCT); t3, 6 months post-randomisation (RCT); t4, 12 months post-randomisation (RCT); t5, 12 months after t0 (total cohort); t6, 24 months after t0 (total cohort). 
depressive disorder, mania/hypomania, GAD, panic disorder, drug use disorder (Composite International Diagnostic Interview Screening Scales-CIDI) ${ }^{49}$ alcohol use disorder (Alcohol Use Disorders Identification Test ${ }^{50}$ ), intermittent explosive disorder, post-traumatic stress disorder, binge-eating behaviour, purging behaviour, psychotic disorder (CIDI) ${ }^{49} 51$ and suicidal thoughts and behaviours (the Self-Injurious Thoughts and Behaviours Interview $^{52}$ ). Moreover, this survey assesses the self-reported quality of health, use of services for emotional or mental health problems, academic attainment and university expectations and adjustment. The e-survey will be administered at the screening $(\mathrm{t} 0)$.

In the RCT, participants will complete online self-report questionnaires (via the Qualtrics platform) and the MINI clinical diagnostic interview, which is administered via the telephone. In both the intervention and control condition, participants are followed up to 12 months post-randomisation. After screening ( $\mathrm{t} 0$ ), the outcome measures are administered at baseline (t1), post-treatment -7 weeks post-randomisation (t2), 6 months (t3) and 12 months post-randomisation (t4). Participants are invited to complete the assessments through emails. In case a participant is not contactable on the first attempt, the research team sends up to two reminder emails within 2 weeks. To booster study adherence, if a participant does not respond to reminders, the research team contacts the participant via telephone. Figure 1 shows the flowchart of participants' inclusion.

\section{Sample size calculation}

The power calculation is based on a head-to-head comparison of the guided web-based transdiagnostic intervention versus TAU (t-test). We have decided to calculate our sample size based on the effects of web-based interventions on depressive symptoms. We have made this choice because web-based interventions have overall higher effects on anxiety compared with depression. Thus, we anticipate a conservative estimate of Cohen's $=0.70$ based on two recent meta-analyses on the effectiveness of psychotherapy in treating depressive symptoms in college students. ${ }^{53}$ If we set the statistical power at 0.80 and alpha at 0.05 , according to a two-tailed hypothesis, we need 34 participants per group to obtain a Cohen's $\mathrm{d}$ of 0.70 (total $n=68$ ). Previous literature has shown that guided web-based interventions have a dropout rate of $28 \%{ }^{55}$ Thus, considering the potential dropouts, the minimum sample for the RCT is 88 participants (44 participants per group).

\section{Recruitment}

Participants are recruited from Dutch universities and colleges. Recruitment for the RCT is conducted through the e-survey of the WHO WMH-ICS. Recruitment for the survey is conducted in two ways: First, we recruit participants through emails and advertisements (eg, flyers, faculty newsletters, social media and university websites). The advertisements target all college students to inform them about the study and emphasise the importance of self-help in improving well-being and academic achievement. We have also created a website for this study (https://caring-universities.com), which contains information and useful links for questions. The research team sends emails to students providing information about the project and a link to the screening questionnaires. A reminder is sent to non-responders biweekly. Students can unsubscribe from the reminder emails whenever they want and their participation is voluntary. Second, study advisors, students' mentors and student ambassadors inform college students about the study.

After completing the e-survey, students eligible for the RCT are notified instantly. Those who are not eligible are sent a thank you email for their participation in the survey. The research team approaches those who have severe symptoms of depression and/or anxiety to inform them about the available treatment options in the community. Students who meet inclusion criteria (as described in the Eligibility criteria section) are informed about the RCT. Those who are interested in participating receive a more detailed information brochure about the study along with an informed consent form. After returning a signed informed consent form, students are invited by email for a telephone MINI diagnostic interview. After the diagnostic interview, students are randomised to either the web-based intervention or the TAU group. After randomisation, students are sent to a link (via email) to the online baseline questionnaires. Students who are assigned to the intervention arm are asked to create an account to follow the web-based intervention. Students in the TAU group receive information about the available regular care services in the community.

\section{Randomisation, blinding and treatment allocation}

Two independent researchers who are not involved in the study generate a random sequence using a computer random sequence generator. Randomisation takes place at an individual level, stratified by recruitment location (the Vrije Universiteit or the Universiteit van Amsterdam). Participants are randomised into two groups (web-based intervention vs TAU) with an allocation ratio of $1: 1$. We conduct block randomisation with randomly varied block sizes (6-12 allocations per block). The allocation is concealed from study's researchers since the randomisation is conducted using a computer-generated code by an independent researcher. It is not possible to mask personnel and participants to the treatment allocation because of the nature of the intervention. However, the MINI diagnostic interview will be performed by blind interviewers with no knowledge about the allocation assignment.

\section{Data collection and management}

This study follows the European Union General Data Protection Regulations. All data are driven from self-report questionnaires and are mostly collected through electronic means (Qualtrics platform). However, according 


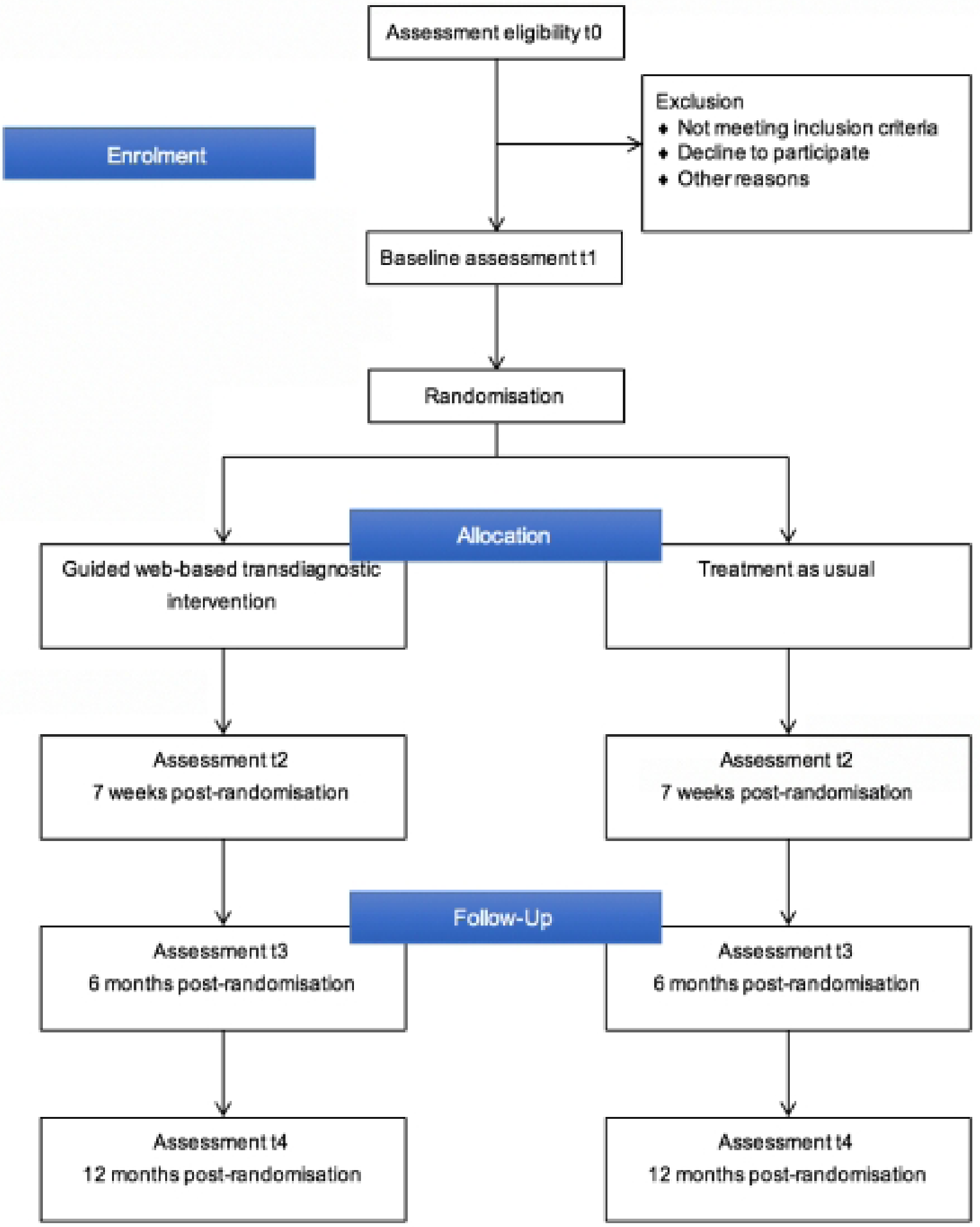

Figure 1 Flowchart of the participants' inclusion process.

to the regulations of the Medical Ethics Committee of the VU Medical Center (VUmc), electronic informed consents are not allowed. Thus, we collect all signed informed consent forms via post. To ensure data confidentiality, participants' informed consent forms are locked in the institution allowing only authorised research staff to have access. Electronic data are password protected in a secure environment and are accessed only by authorised personnel. The primary use of the data is anonymous.

\section{Statistical analysis}

All randomised participants will be included in all analyses according to the intention-to-treat principle. Missing values will be imputed using multiple imputations. Also, 
we will conduct per protocol analyses including only those who completed post-treatment and follow-up assessments. All analyses will be performed using STATA V.SE 13.1. ${ }^{56}$ The results of the MINI interview will be summarised using descriptive statistics. We will analyse the effects of the interventions on depression (PHQ-9) and anxiety severity (GAD-7) at both post-treatment and follow-up assessment using multilevel mixed models linear regression with a restricted maximum likelihood algorithm. The post-treatment depression and anxiety scores will be used as a dependent variable and trial arm condition (web-based transdiagnostic individually tailored intervention vs TAU) as an independent variable while adjusting for baseline depression and anxiety severity. Additionally, we will calculate the effect size, Cohen's d, by subtracting the average score on primary outcome measures (PHQ-9 and GAD-7 scales) of the intervention group from the average scores of the control group at the post-treatment and dividing the results by the pooled SD. To measure clinical significance, we will calculate response and symptom deterioration rates according to the Reliable Change Index. ${ }^{57}$ The reliable change will be calculated using the pretreatment $\mathrm{SD}$, and the test-retest reliability coefficient of PHQ-9 $(0.76)^{31}$ and GAD-7 (0.83). ${ }^{39}$ Analogously, Cohen's d and clinically significant change will be calculated for follow-up assessments (6 and 12 months). Finally, at the 12-month follow-up assessment, we will analyse the effects of the intervention on the current diagnosis of depression and anxiety disorders, using the data from MINI. We will use a multilevel mixed-effects logistic regression with a restricted maximum likelihood algorithm. The current diagnosis of depression or anxiety disorders will be used as a dependent variable and trial arm condition as independent while adjusting for baseline depression and anxiety severity.

\section{Patient and public involvement}

Student representatives and college/university stakeholders (eg, student psychological counsellors, study advisors, deans of education, deans of the faculties, the university executive board members, etc) were involved in various stages of the development of the intervention and the trial. Before the development of the protocol, the research team had several discussions with student representatives and university stakeholders regarding their views about this study. These discussions aimed at gaining a better understanding of the end-users and stakeholders' perspective, needs and preferences to inform the development of the study procedures. Moreover, we performed several focus group discussions to tailor the intervention to the college/universitystudent context. Finally, student representatives participated in brainstorming discussions regarding the design of the best recruitment strategy.

\section{Possible harms}

According to the previous literature, Internet-based interventions lead to lower symptom deterioration rates compared with controls. ${ }^{58} 59$ Moreover, in this study, participants are college students with mild to moderate symptoms of depression and/or anxiety. This population has a high degree of functioning (eg, attending university) and is unlikely to enter the general medical healthcare system. Nevertheless, psychological intervention might lead to unwanted outcomes. For instance, it is possible that the students experience suicidal ideation. If we detect a student who is at high suicidal risk, a specific protocol is followed: the e-coach calls the student to assess the risk by asking a series of questions. Afterwards, the e-coach contacts an experienced psychiatrist, who is involved in the study, to discuss the situation. If needed, the psychiatrist contacts the participant to advise him/her to seek help from his/her GP or the student counselling services. The research team checks if the student sought help after a couple of days. Moreover, if the student permits us to use the contact details of his/herGP, the research team notifies the GP to ensure that the student will get help timely.

Adverse events (eg, increase suicidal risk, hospital admission, clinically significant symptom deterioration, and study and treatment dropout rates) will be monitored and recorded throughout the trial. All adverse events will be reported per group in the outcomes of the present study.

\section{Premature termination of the study}

The research team will decide to terminate the ongoing trial in case of serious adverse events (eg, suicide), which is directly related to the study procedures. The principal investigator (PC) will prompt the discontinuation of the trial and will inform the Medical Ethics Committee immediately. All participants will be informed about the study termination and the reason that led to this decision. Moreover, participants will receive information about available mental healthcare services options

\section{Ethics}

The study will be conducted following the principles of the Declaration of Helsinki (64th World Medical Association General Assembly, Fortaleza, Brazil, October 2013) and in accordance with the Medical Research Involving Human Subjects Act. The Medical Ethics Committee of the VUmc monitors the progress and procedures of the trial.

\section{DISCUSSION}

Early management of depression and anxiety may improve symptoms, increase academic performance and prevent college dropout. The present protocol describes the procedures of an RCT conducted in Dutch universities and colleges. This study aims at examining the effects of a guided transdiagnostic individually tailored web-based intervention in reducing symptoms of depression and/ or anxiety in college student population. It is expected that the examined intervention will outperform TAU in treating college students with depression and/or anxiety. 
So far, only a few trials have examined the effectiveness of web-based transdiagnostic and individually tailored interventions in college students. The outcomes of these trials were mixed and thus, inconclusive. ${ }^{10}$ Moreover, to the best of our knowledge, previous studies on college students' mental health have mostly focused on one disorder. Given that depression and anxiety are highly comorbid, ${ }^{60-62}$ it is essential to test approaches with transdiagnostic components targeted at symptoms of both depression and anxiety. The present study aims at improving existing knowledge on the effectiveness of web-based interventions in college students suffering from depression and/or anxiety by employing a transdiagnostic and individually tailored therapeutic approach. This study targets both Dutch and international students, thereby increasing the generalisability of our findings to different cultural backgrounds.

Nevertheless, several limitations should be expected. First, the power calculation has been based on our primary aim to examine the effectiveness of the web-based transdiagnostic individually tailored intervention in reducing symptoms of depression and/or anxiety. Therefore, the study is underpowered to examine secondary moderator analyses, which usually require larger sample sizes. If possible, we will recruit a larger number of participants to achieve sufficient power for secondary outcomes such as college dropout, as well as the planned moderator analysis. Second, although the intervention is delivered with therapeutic guidance, retaining students in the intervention might be a challenge. However, dropout has been considered in sample size calculation and thus, we expect that it will not influence the statistical power of our sample. Third, we cannot measure educational achievement using academic records due to ethical restrictions. Information on educational attainment will be self-reported and thus, may be less objective.

Overall, the results of this study will provide valuable information about the effectiveness of web-based interventions in improving college students' mental health and may lead to the development of the infrastructure for screening and treating mental disorders within universities.

\section{TRIAL STATUS}

The trial has started in March 2018 and is expected to be completed in August 2019.

\section{Author affiliations}

${ }^{1}$ Department of Clinical, Neuro, and Developmental Psychology, Vrije Universiteit Amsterdam, Amsterdam, The Netherlands

${ }^{2}$ Amsterdam Public Health Research Institute, Faculty of Behavioural and Movement Sciences, Vrije Universiteit Amsterdam, Amsterdam, The Netherlands

${ }^{3}$ Addiction, Development, and Psychopathology Lab, Department of Psychology, University of Amsterdam, Amsterdam, The Netherlands

${ }^{4}$ Clinical Psychology and Psychotherapy, Friedrich-Alexander-University ErlangenNuremberg, Erlangen, Germany

${ }^{5}$ Department of Psychiatry, Columbia University, New York, New York, USA ${ }^{6}$ Department of Health Care Policy, Harvard Medical School, Boston, Massachusetts, USA
${ }^{7}$ Universitair Psychiatrisch Centrum, Centre for Public Health Psychiatry, Katholieke Universiteit Leuven, Leuven, Belgium

${ }^{8}$ Department of Psychiatry and Department of Epidemiology and Biostatistics, VU University Medical Centre Amsterdam, Amsterdam Public Health Research Institute, Amsterdam, The Netherlands

${ }^{9}$ Department of Epidemiology and Biostatistics, VU University Medical Centre Amsterdam, Amsterdam Public Health Research Institute, Amsterdam, The Netherlands

${ }^{10}$ Department of Research, Development and Prevention, Student Health Service University of Amsterdam, Amsterdam, The Netherlands

Acknowledgements We thank all the student representatives as well as the university stakeholders of the Vrije Universiteit (VU) of Amsterdam and the Universiteit van Amsterdam (UvA) for their great help during the development of our study.

Contributors PC (PI), HR and RWW obtained funding for this study. All authors contributed to the design of the study. EK drafted the manuscript and coordinated the recruitment of students and the data collection at VU. AMK coordinates the recruitment of students and the data collection at UvA. RWW, HR, LdW, AMK, EB, SB, $\mathrm{FB}, \mathrm{NB}, \mathrm{CMvdH}, \mathrm{PV}, \mathrm{AK}, \mathrm{LK}, \mathrm{DDE}, \mathrm{RB}, \mathrm{RCK}, \mathrm{RPA}$ and $\mathrm{PC}$ were involved in revising the manuscript critically for intellectual content. All authors read and approved the final manuscript.

Funding This trial is funded by ZonMw, Research Program GGz, grant number 636110005 .

Competing interests In the past 3 years, Dr. Kessler received support for his epidemiological studies from Sanofi Aventis; was a consultant for Johnson \& Johnson Wellness and Prevention, Sage Pharmaceuticals, Shire, Takeda; and served on an advisory board for the Johnson \& Johnson Services Inc. Lake Nona Life Project. Kessler is a co-owner of DataStat, Inc., a market research firm that carries out healthcare research.

Patient consent for publication Not required.

Ethics approval The Medical Ethics Committee of the VUmc has approved the protocol (registration number 2016.583 and A2017.362) and all the amendments will be notified to this committee.

Provenance and peer review Not commissioned; externally peer reviewed.

Open access This is an open access article distributed in accordance with the Creative Commons Attribution Non Commercial (CC BY-NC 4.0) license, which permits others to distribute, remix, adapt, build upon this work non-commercially, and license their derivative works on different terms, provided the original work is properly cited, appropriate credit is given, any changes made indicated, and the use is non-commercial. See: http://creativecommons.org/licenses/by-nc/4.0/.

\section{REFERENCES}

1. Alonso J, Vilagut G, Mortier P, et al. The role impairment associated with mental disorder risk profiles in the WHO World Mental Health International College Student Initiative. Int J Methods Psychiatr Res 2018:e1750 (published Online First: 2018/11/08).

2. Auerbach RP, Mortier P, Bruffaerts R, et al. WHO World Mental Health Surveys International College Student Project: Prevalence and distribution of mental disorders. J Abnorm Psychol 2018;127:623-38.

3. Reavley N, Jorm AF. Prevention and early intervention to improve mental health in higher education students: a review. Early Interv Psychiatry 2010;4:132-42.

4. Buchanan JL. Prevention of depression in the college student population: a review of the literature. Arch Psychiatr Nurs 2012;26:21-42.

5. Auerbach RP, Alonso J, Axinn WG, et al. Mental disorders among college students in the World Health Organization World Mental Health Surveys. Psychol Med 2016;46:2955-70.

6. Hysenbegasi A, Hass SL, Rowland CR. The impact of depression on the academic productivity of university students. $J$ Ment Health Policy Econ 2005;8:145-51.

7. Kessler RC, Foster CL, Saunders WB, et al. Social consequences of psychiatric disorders, I: Educational attainment. Am J Psychiatry 1995;152:1026-32.

8. Blanco C, Okuda M, Wright C, et al. Mental health of college students and their non-college-attending peers: results from the National Epidemiologic Study on Alcohol and Related Conditions. Arch Gen Psychiatry 2008;65:1429-37. 
9. Cuijpers P, Auerbach RP, Benjet C, et al. The World Health Organization World Mental Health International College Student initiative: An overview. Int J Methods Psychiatr Res 2019:e1761 (published Online First: 2019/01/08).

10. Farrer L, Gulliver A, Chan JK, et al. Technology-based interventions for mental health in tertiary students: systematic review. $J$ Med Internet Res 2013;15:e101.

11. Hunt J, Eisenberg D. Mental health problems and help-seeking behavior among college students. J Adolesc Health 2010;46:3-10.

12. Van der Heijde CM, Vonk P, Meijman FJ. Self-regulation for the promotion of student health. Traffic lights: the development of a tailored web-based instrument providing immediate personalized feedback. Health Psychol Behav Med 2015;3:169-89.

13. Josephine K, Josefine L, Philipp D, et al. Internet- and mobilebased depression interventions for people with diagnosed depression: A systematic review and meta-analysis. J Affect Disord 2017;223:28-40.

14. Karyotaki E, Ebert DD, Donkin L, et al. Do guided internet-based interventions result in clinically relevant changes for patients with depression? An individual participant data meta-analysis. Clin Psychol Rev 2018;63:80-92

15. Karyotaki E, Riper H, Twisk J, et al. Efficacy of Self-guided InternetBased Cognitive Behavioral Therapy in the Treatment of Depressive Symptoms: A Meta-analysis of Individual Participant Data. JAMA Psychiatry 2017;74:351-9.

16. Domhardt M, Geßlein H, von Rezori RE, et al. Internet- and mobilebased interventions for anxiety disorders: A meta-analytic review of intervention components. Depress Anxiety 2019;36:213-24.

17. Ebert DD, Van Daele T, Nordgreen T, et al. Internet- and MobileBased Psychological Interventions: Applications, Efficacy, and Potential for Improving Mental Health. Eur Psychol 2018;23:167-87.

18. Andersson G, Cuijpers P, Carlbring P, et al. Guided Internet-based vs face-to-face cognitive behavior therapy for psychiatric and somatic disorders: a systematic review and meta-analysis. World Psychiatry 2014;13:288-95.

19. Berger T, Boettcher J, Caspar F. Internet-based guided selfhelp for several anxiety disorders: a randomized controlled trial comparing a tailored with a standardized disorder-specific approach. Psychotherapy 2014;51:207-19.

20. Carlbring P, Maurin L, Törngren C, et al. Individually-tailored, Internetbased treatment for anxiety disorders: A randomized controlled trial. Behav Res Ther 2011;49:18-24.

21. Silfvernagel K, Carlbring P, Kabo J, et al. Individually tailored internetbased treatment for young adults and adults with panic attacks: randomized controlled trial. J Med Internet Res 2012;14:e65.

22. Titov N, Dear BF, Schwencke G, et al. Transdiagnostic internet treatment for anxiety and depression: a randomised controlled trial. Behav Res Ther 2011;49:441-52.

23. Păsărelu CR, Andersson G, Bergman Nordgren L, et al. Internetdelivered transdiagnostic and tailored cognitive behavioral therapy for anxiety and depression: a systematic review and meta-analysis of randomized controlled trials. Cogn Behav Ther 2017:46:1-28.

24. Harrer M, Adam SH, Baumeister $\mathrm{H}$, et al. Internet interventions for mental health in university students: A systematic review and metaanalysis. Int J Methods Psychiatr Res 2018;13:e1759.

25. Braithwaite SR, Fincham FD. ePREP: Computer Based Prevention of Relationship Dysfunction, Depression and Anxiety. J Soc Clin Psychol 2007;26:609-22.

26. Cukrowicz KC, Joiner TE. Computer-Based Intervention for Anxious and Depressive Symptoms in a Non-Clinical Population. Cognit Ther Res 2007;31:677-93.

27. Seligman ME, Schulman P, Tryon AM. Group prevention of depression and anxiety symptoms. Behav Res Ther 2007;45:1111-26.

28. Kenardy J, McCafferty K, Rosa V. Internet-Delivered Indicated Prevention For Anxiety Disorders: A Randomized Controlled Trial. Behav Cogn Psychother 2003;31:279-89.

29. Day V, McGrath PJ, Wojtowicz M. Internet-based guided self-help for university students with anxiety, depression and stress: a randomized controlled clinical trial. Behav Res Ther 2013;51:344-51 https://doi. org/.

30. Mullin A, Dear BF, Karin E, et al. The UniWellbeing course: A randomised controlled trial of a transdiagnostic internet-delivered cognitive behavioural therapy (CBT) programme for university students with symptoms of anxiety and depression. Internet Interv 2015;2:128-36.

31. Kroenke K, Spitzer RL, Williams JB. The PHQ-9: validity of a brief depression severity measure. J Gen Intern Med 2001;16:606-13.
32. Spitzer RL, Kroenke K, Williams JB, et al. A brief measure for assessing generalized anxiety disorder: the GAD-7. Arch Intern Med 2006;166:1092-7.

33. Sheehan DV, Lecrubier Y, Sheehan KH, et al. The Mini-International Neuropsychiatric Interview (M.I.N.I.): the development and validation of a structured diagnostic psychiatric interview for DSM-IV and ICD-10. J Clin Psychiatry 1998;59 Suppl 20(Suppl 20):22-33.

34. Weisel KK, Zarski AC, Berger T, et al. Efficacy and cost-effectiveness of guided and unguided internet- and mobile-based indicated transdiagnostic prevention of depression and anxiety (ICare Prevent): A three-armed randomized controlled trial in four European countries. Internet Interv 2019;16.

35. Weisel KK, Zarski AC, Berger T, et al. Transdiagnostic Tailored Internet- and Mobile-Based Guided Treatment for Major Depressive Disorder and Comorbid Anxiety: Study Protocol of a Randomized Controlled Trial. Front Psychiatry 2018;9.

36. Bolinski F, Kleiboer A, Karyotaki E, et al. Effectiveness of a transdiagnostic individually tailored Internet-based and mobilesupported intervention for the indicated prevention of depression and anxiety (ICare Prevent) in Dutch college students: study protocol for a randomised controlled trial. Trials 2018;19:118.

37. Kroenke K, Spitzer RL. The PHQ-9: A New Depression Diagnostic and Severity Measure. Psychiatr Ann 2002;32:509-15.

38. Wittkampf KA, Naeije L, Schene AH, et al. Diagnostic accuracy of the mood module of the Patient Health Questionnaire: a systematic review. Gen Hosp Psychiatry 2007;29:388-95.

39. Dear BF, Titov N, Sunderland M, et al. Psychometric comparison of the generalized anxiety disorder scale-7 and the Penn State Worry Questionnaire for measuring response during treatment of generalised anxiety disorder. Cogn Behav Ther 2011;40:216-27.

40. Lecrubier Y, Sheehan DV, Weiller E, et al. The Mini International Neuropsychiatric Interview (MINI). A short diagnostic structured interview: reliability and validity according to the CIDI. European Psychiatry 1997;12:224-31.

41. Group E. EuroQol - a new facility for the measurement of healthrelated quality of life. Health Policy 1990;16:199-208.

42. van Agt HM, Essink-Bot ML, Krabbe PF, et al. Test-retest reliability of health state valuations collected with the EuroQol questionnaire. Soc Sci Med 1994;39:1537-44.

43. König $\mathrm{H}-\mathrm{H}$, Heider D, Lehnert T, et al. Health status of the advanced elderly in six European countries: results from a representative survey using EQ-5D and SF-12. Health and quality of life outcomes 2010;8:143

44. Larsen DL, Attkisson CC, Hargreaves WA, et al. Assessment of client/patient satisfaction: development of a general scale. Eval Program Plann 1979;2:197-207.

45. Attkisson CC, Greenfield TK. The Client Satisfaction Questionnaire (CSQ) Scales and the Service Satisfaction Scale-30 (SSS-30). In: Sederer LI, Dickey B, eds. Outcomes assessment in clinical practice. Baltimore, MD: Williams \& Wilkins, 1996:120-7.

46. Boß L, Lehr D, Reis D, et al. Reliability and Validity of Assessing User Satisfaction With Web-Based Health Interventions. J Med Internet Res 2016;18:e234-e34.

47. Matsushita M, Adachi $\mathrm{H}$, Arakida M, et al. Presenteeism in college students: reliability and validity of the Presenteeism Scale for Students. Qual Life Res 2011;20:439-46.

48. Kessler RC, Adler L, Ames M, et al. The World Health Organization Adult ADHD Self-Report Scale (ASRS): a short screening scale for use in the general population. Psychol Med 2005;35:245-56.

49. Kessler RC, Ustün TB. The World Mental Health (WMH) Survey Initiative Version of the World Health Organization (WHO) Composite International Diagnostic Interview (CIDI). Int J Methods Psychiatr Res 2004:13:93-121.

50. Saunders JB, Aasland OG, Babor TF, et al. Development of the Alcohol Use Disorders Identification Test (AUDIT): WHO Collaborative Project on Early Detection of Persons with Harmful Alcohol Consumption-II. Addiction 1993;88:791-804.

51. Kessler RC, Santiago PN, Colpe LJ, et al. Clinical reappraisal of the Composite International Diagnostic Interview Screening Scales (CIDI-SC) in the Army Study to Assess Risk and Resilience in Servicemembers (Army STARRS). Int $J$ Methods Psychiatr Res 2013;22:303-21.

52. Nock MK, Holmberg EB, Photos VI, et al. Self-Injurious Thoughts and Behaviors Interview: development, reliability, and validity in an adolescent sample. Psychol Assess 2007;19:309-17.

53. Davies EB, Morriss R, Glazebrook C. Computer-delivered and web-based interventions to improve depression, anxiety, and psychological well-being of university students: a systematic review and meta-analysis. J Med Internet Res 2014;16:e130. 
54. Cuijpers P, Cristea IA, Ebert DD, et al. Psychological Treatment of Depression in College Students: a Metaanalysis. Depress Anxiety 2016;33:400-14.

55. Richards D, Richardson T. Computer-based psychological treatments for depression: a systematic review and meta-analysis. Clin Psychol Rev 2012;32:329-42.

56. Stata Statistical Software: Release 13 [program]. College Station, TX: StataCorp LP 2015.

57. Jacobson NS, Truax P. Clinical significance: a statistical approach to defining meaningful change in psychotherapy research. $J$ Consult Clin Psychol 1991;59:12-19.

58. Karyotaki E, Kemmeren L, Riper $\mathrm{H}$, et al. Is self-guided internetbased cognitive behavioural therapy (iCBT) harmful? An individual participant data meta-analysis. Psychol Med 2018;48:2456-66.
59. Ebert DD, Donkin L, Andersson G, et al. Does Internet-based guidedself-help for depression cause harm? An individual participant data meta-analysis on deterioration rates and its moderators in randomized controlled trials. Psychol Med 2016;46:2679-93.

60. Hirschfeld RM. The Comorbidity of Major Depression and Anxiety Disorders: Recognition and Management in Primary Care. Prim Care Companion J Clin Psychiatry 2001;3:244-54.

61. Kessler RC, Berglund P, Demler O, et al. Lifetime prevalence and age-of-onset distributions of DSM-IV disorders in the National Comorbidity Survey Replication. Arch Gen Psychiatry 2005;62:593-602.

62. Kessler RC, Birnbaum HG, Shahly V, et al. Age differences in the prevalence and co-morbidity of DSM-IV major depressive episodes: results from the WHO World Mental Health Survey Initiative. Depress Anxiety 2010;27:351-64. 